\title{
Eigenvalue conjecture and colored Alexander polynomials
}

\author{
A. Mironov ${ }^{1,2,3,4, a}$, A. Morozov $2,3,4, b$ \\ ${ }^{1}$ Lebedev Physics Institute, Moscow 119991, Russia \\ ${ }^{2}$ ITEP, Moscow 117218, Russia \\ ${ }^{3}$ Institute for Information Transmission Problems, Moscow 127994, Russia \\ ${ }^{4}$ National Research Nuclear University MEPhI, Moscow 115409, Russia
}

Received: 19 October 2016 / Accepted: 26 March 2018 / Published online: 5 April 2018

(C) The Author(s) 2018

\begin{abstract}
We connect two important conjectures in the theory of knot polynomials. The first one is the property $A l_{R}(q)=A l_{[1]}\left(q^{|R|}\right)$ for all single hook Young diagrams $R$, which is known to hold for all knots. The second conjecture claims that all the mixing matrices $U_{i}$ in the relation $\mathcal{R}_{i}=U_{i} \mathcal{R}_{1} U_{i}^{-1}$ between the $i$ th and the first generators $\mathcal{R}_{i}$ of the braid group are universally expressible through the eigenvalues of $\mathcal{R}_{1}$. Since the above property of Alexander polynomials is very well tested, this relation provides new support to the eigenvalue conjecture, especially for $i>2$, when its direct check by evaluation of the Racah matrices and their convolutions is technically difficult.
\end{abstract}

\section{Introduction}

An indisputable advantage of knot theory from the point of view of representation theory is that the former provides a set of quantities that adequately capture and reveal the basic hidden properties of the latter. These quantities, knot polynomials [1-8] are the most natural in quantum field theory (QFT) realization of knot theory: they are just the Wilson loop averages in the topological Chern-Simons model $[9,10]$, which is one of the simplest in the family of Yang-Mills theories. The power of knot polynomial QFT methods in knot and representation theories is an impressive manifestation of the effectiveness of string theory approach to mathematical problems, especially when their calculational (algebraic) aspects are concerned.

This letter is an attempt to "explain" the amusing property of Alexander polynomials, the specializations of the (colored) HOMFLY polynomials at $A=1$,

$A l_{R}^{\mathcal{K}}(q):=H_{R}^{\mathcal{K}}(A=1, q)$.

\footnotetext{
a e-mails: mironov@1pi.ru; mironov@itep.ru

b e-mail: morozov@itep.ru
}

The property is that

$A l_{R}^{\mathcal{K}}(q)=A l_{\square}^{\mathcal{K}}\left(q^{|R|}\right)$

for all knots $\mathcal{K}$ and all single hook Young diagrams $R=$ $\left[r, 1^{s}\right]$ of size $|R|=r+s$. This phenomenon was "experimentally" observed in [11] (and later discussed in [12]) as a "dual" to the property of special polynomials [13] (discussed in $[12,14]$ and generalized to superpolynomials in [15-17]), arising from the HOMFLY polynomial at $q=1$,

$H_{R}^{\mathcal{K}}(A, q=1)=\left(H_{\square}^{\mathcal{K}}(A, q=1)\right)^{|R|}$,

which is in fact valid for all Young diagrams $R$ (not obligatory single hook), and which was actually proved rather fast [14]. Equation (2), however, got less attention and still remains a mystery. In the present text, we reduce it to another "experimental" discovery, the eigenvalue conjecture of [18], moreover, (2) follows from its stronger version, applicable to arbitrary number $m$ of strands in the braid (the weak form considered in most detail in [18] concerned only $m=3$ and the ordinary Racah matrices).

Namely, we decompose the explanation of (2) into five steps:

- Realize the knot $\mathcal{K}$ as a closure of an $m$-strand braid; a lot of such realizations is possible for any $\mathcal{K}$, equivalence of the HOMFLY polynomials for different choices is guaranteed by invariance under the Reidemeister moves. Then (see [13,19-40])

$H_{R}^{\mathcal{K}}(A, q)=\sum_{Q \vdash m|R|} C_{R Q}(q) \cdot \frac{\chi_{Q}^{*}}{\chi_{R}^{*}}(A, q)$

where all the dependence on $A$ is localized in the quantum dimensions ( $q$-graded traces) $\chi^{*}$. 
- The quantum dimensions are given by the (hook) product formula over all boxes of the Young diagram:

$\chi_{Q}^{*}=\prod_{\square \in Q} \frac{\left\{A q^{l_{\square}^{\prime}-a_{\square}^{\prime}}\right\}}{\left\{q^{l_{\square}+a_{\square}+1}\right\}}$

where $\{x\}=x-x^{-1}$, while $l, a, l^{\prime}, a^{\prime}$ are the lengths of legs, arms, co-legs and co-arms, respectively.

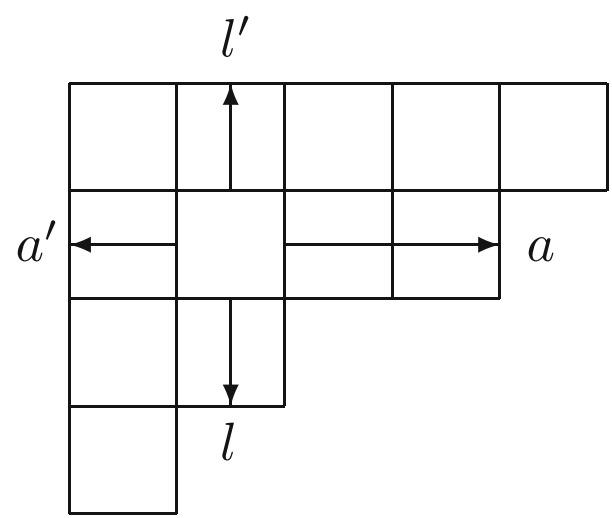

Contributing to (4) at $A=1$ are diagrams $Q$ with no $l=a$ factors (which vanish at $A=1$ ). One such factor is obligatory present for any $Q$, but it drops away from the ratio $\chi_{Q}^{*} / \chi_{R}^{*}$. For a single hook $R=\left[r, 1^{s}\right]$, however, contributing $Q$ are also only single hook. Since their sizes are $|Q|=m|R|=$ $m(r+s)$, and at the same time the numbers of rows and columns are restricted by $m r$ and $m(s+1)$, respectively, the set of $Q$ contributing to (4) at $A=1$ consists just of $m$ single hook diagrams,

$Q=\left[m r-k, 1^{m s+k}\right], \quad k=0, \ldots, m-1$.

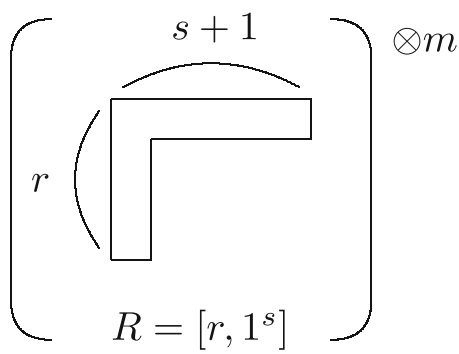

$$
C_{R Q}=\operatorname{Tr}_{V_{Q}}\left(\mathcal{R}_{1}^{a_{1,1}} \ldots \mathcal{R}_{m-1}^{a_{1, m-1}} \mathcal{R}_{1}^{a_{2,1}} \ldots \mathcal{R}_{m-1}^{a_{2, m-1}} \ldots\right)
$$

Here the trace is taken over the space of intertwining operators (multiplicities), $R^{\otimes m}=\oplus_{Q} V_{Q}^{(m)} \otimes Q$ and the $\mathcal{R}$-matrix $\mathcal{R}_{i}$ standing at the intersection of $i$ th strand with the $(i+1)$ th one is obtained by the conjugation

$\mathcal{R}_{i}=U_{i} \mathcal{R}_{1} U_{i}^{-1}$

It is associated with the fact that the usual $\mathcal{R}$-matrix which acts in the space of product of two representations $R \otimes R$ if diagonalized, is proportional to unity in the space of the irreducible representation $Y$ that appears in the decomposition of the square of $R, R \otimes R=\oplus_{Y} V_{Y}^{(2)} \otimes Y$ :

$\mathcal{R}_{i}=\oplus_{Y} \epsilon_{i} q^{\varkappa_{Y}} \cdot I_{V_{Y}^{(2)}}$

where $\epsilon_{i}= \pm 1$, depending on whether $Y$ belongs to the symmetric or antisymmetric square.

This is why one can work with $\mathcal{R}$, which acts already in the space of intertwining operators $V_{Y}^{(2)}$. One can diagonalize, say, $\mathcal{R}_{1}$ with acts on the first two strands. However, all matrices $\mathcal{R}_{i}$ cannot be diagonalized at once. For $m>2$ each multiplicity space $V_{Q}^{(2)}$, arising in the $m$ th tensor power of $R$, contains descendants of different $Y$ from the second level, and while $\mathcal{R}_{1}$ remains diagonal, the other $\mathcal{R}_{i}$ (and thus elementary building blocks of $U_{i}[35,37]$ ) after a proper ordering of columns and rows are block-diagonal matrices with blocks of the size $V_{Q}^{(2)} \otimes V_{Q}^{(2)}[35,37,38]$.

If both $R$ and $Q \in R^{\otimes m}$ are single hook diagrams, then $Y \in R^{\otimes 2}$, lying on the path from $R$ to $Q$ in the representation graph [38], are also single hook, and there are just two of the present, those with $k=0$ and $k=1$. Up to a common $R$-dependent shift, the eigenvalues (9) are equal to

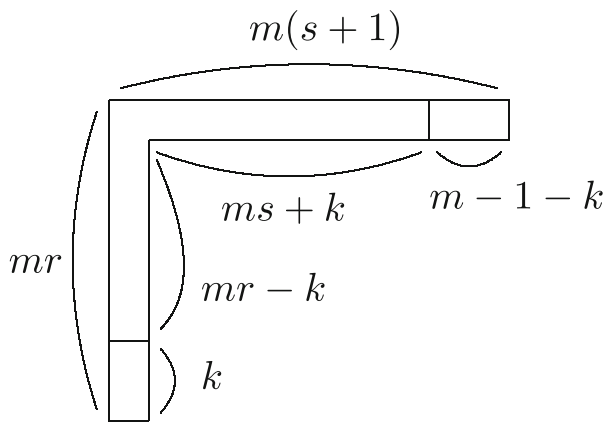

a single hook $Q=\left[m r-k, 1^{m s+k}\right] \in R^{\otimes m}$

- If the knot $\mathcal{K}$ is realized as a closure of the $m$-strand braid $\left(a_{1,1}, \ldots, a_{1, m-1}\left|a_{2,1}, \ldots a_{2, m-1}\right| \ldots\right)$, then the coefficients $C_{R Q}$ in (4) are actually equal to [25-40]

$\varkappa_{Y}=\operatorname{shift}+\sum_{(i, j) \in Y}(i-j) \stackrel{(6)}{=} \operatorname{shift} \pm \underbrace{(r+s)}_{|R|}$ 
for $Y=\left[2 r, 1^{2 s}\right]$ and $Y=\left[2 r-1,1^{2 s+1}\right]$. In other words, the difference between eigenvalues for representation/Young diagram $R$ and for the fundamental representation $\square$ is exactly in the $|R|$ th power of $q$, the same as in (2) (this is since the common shift is irrelevant: it is the same in the two cases in the topological framing).

- It is a simple exercise to check that exactly the same is true for the ratios of quantum dimensions $\chi_{Q}^{*} / \chi_{R}^{*}$ :

$$
\left.\frac{\chi_{\left[r m-k, 1^{m s+k}\right]}^{*}}{\chi_{\left[r, 1^{s}\right]}^{*}}\right|_{A=1}=\frac{[r+s]}{[m(r+s)]}=\frac{[|R|]}{[m|R|]}
$$

where the quantum numbers are defined as $[N]=\frac{\left\{q^{N}\right\}}{\{q\}}=$ $\frac{q^{N}-q^{-N}}{q-q^{-1}}$.

Keeping all this in mind, for the $m=2$-strand knots with odd $n$, one obtains from (4)

$$
\begin{gathered}
A l_{\left[r, 1^{s}\right]}^{(n)}=\frac{[r+s]}{[2(r+s)]} \cdot\left(q^{n(r+s)}+q^{-n(r+s)}\right) \\
=\frac{q^{n(r+s)}+q^{-n(r+s)}}{q^{r+s}+q^{-(r+s)}}=\frac{q^{n|R|}+q^{-n|R|}}{q^{|R|}+q^{-|R|}}
\end{gathered}
$$

which for odd $n$ (i.e. for the 2-strand knots rather than links) is indeed a polynomial, satisfying (2).

- For $m>2$ just the same reasoning would work with the only correction: the mixing matrices $U$ emerge. For (2) to be true, it is sufficient if $U$-matrices depend on $R=\left[r, 1^{s}\right]$ through $q^{r+s}=q^{|R|}$ only - and this is exactly what follows from the eigenvalue conjecture of [18]. The conjecture claims that the $V_{Q} \otimes V_{Q}$ block of $U$, associated with representation $Q$, are made entirely from the normalized eigenvalues $\epsilon_{Y} q^{\varkappa_{Y}}$ of the $\mathcal{R}$-matrix $\mathcal{R}_{1}$ for $Y$ on the path from $R$ to $Q$, and (10) shows that in our single hook case these are in turn made exactly from $q^{r+s}=q^{|R|}$ ("normalized" means that the "shifts" in (10) can be neglected).

It is, of course, important in this argument that the paths in representation graph from a single hook $R$ to a single hook $Q$ are restricted to single hook diagrams at all steps. As a result, the encountered diagrams and, thus, the entire paths are labeled by the parameters $k$, which are actually $R$ independent: the paths and contributing eigenvalues are just the same for the fundamental representation and for any other single hook $R$.

\section{Thus, the eigenvalue conjecture implies (2).}

This is the main claim of the present letter. However, in practice this statement is evidence in favor of the eigenvalue conjecture rather than of (2). This is because (2) is very easy to check, once colored HOMFLY is known, and recent advances in HOMFLY calculus [41-53] provided quite a number of examples, which allows one to consider (2) rather well tested. The situation with the eigenvalue conjecture is much worse: it was quite difficult to check it for the matrix sizes (dimensions of $W(Q)$ ) 2,3,4,5 even in the simplest case of $m=3$ strands. For size 6 it was validated very recently within the framework of the knot universality of $[54,55]$ and by application to advanced Racah calculus in $[46,48,49]$. This was an important step, because, beginning from the size 6 , the eigenvalue conjecture does not immediately follow from the Yang-Baxter relations only [18,56]; still for knot calculus it works well. Evidence for the eigenvalue hypotheses for a higher number of strands $m>3$ is still nearly negligible. Mixing matrices are now not just Racah matrices but their convolutions [57,58], which are extremely difficult to calculate, and not much has been yet done since [25-40]. In this text we presented the claim that, if true for all $m$, the eigenvalue conjecture would explain the well-established equation (2), and this is new and reasonably strong evidence. It is far from proving anything, both because (2) is not proved and because the eigenvalue conjecture is sufficient, but not necessary for (2) to hold. Still, this new relation between the two conjectures should attract new attention to both of them and hopefully lead to a considerably better understanding.

From this interpretation of (2) it becomes clear what is so special about the single hook diagrams. For $R$ with $h$ hooks contributing to the sum (4) at $A=1$ will also be the $h$ hook diagrams $Q$, which will be parameterized by $2 h-1$ parameters instead of a single $k$. Analysis of this situation is now possible and straightforward, however, it is not a surprise that the answer is more sophisticated than (2).

Acknowledgements This work was performed at the Institute for Information Transmission Problems with the financial support of the Russian Science Foundation (Grant no. 14-50-00150).

Open Access This article is distributed under the terms of the Creative Commons Attribution 4.0 International License (http://creativecomm ons.org/licenses/by/4.0/), which permits unrestricted use, distribution, and reproduction in any medium, provided you give appropriate credit to the original author(s) and the source, provide a link to the Creative Commons license, and indicate if changes were made. Funded by SCOAP S $^{3}$

\section{References}

1. J.W. Alexander, Trans. Am. Math. Soc. 30(2), 275-306 (1928)

2. J.H. Conway, Algebraic Properties, In: John Leech (ed.) Computational problems in abstract algebra, Proc. Conf. Oxford, 1967. Pergamon, Oxford, New York, pp 329-358 (1970)

3. V.F.R. Jones, Invent. Math. 72, 1 (1983)

4. V.F.R. Jones, Bull. AMS 12, 103 (1985)

5. V.F.R. Jones, Ann. Math. 126, 335 (1987)

6. L. Kauffman, Topology 26, 395 (1987) 
7. P. Freyd, D. Yetter, J. Hoste, W.B.R. Lickorish, K. Millet, A. Ocneanu, Bull. AMS. 12, 239 (1985)

8. J.H. Przytycki, K.P. Traczyk, Kobe J. Math. 4, 115-139 (1987)

9. S.-S. Chern, J. Simons, Ann. Math. 99, 48-69 (1974)

10. E. Witten, Comm. Math. Phys. 121, 351-399 (1989)

11. H. Itoyama, A. Mironov, A. Morozov, A. Morozov, JHEP 2012, 131 (2012). arXiv: 1203.5978

12. S. Zhu. arXiv: 1206.5886

13. P. Dunin-Barkowski, A. Mironov, A. Morozov, A. Sleptsov, A. Smirnov, JHEP 03, 021 (2013). arXiv: 1106.4305

14. K. Liu, P. Peng. arXiv:0704.1526

15. I. Cherednik, arXiv: 1111.6195

16. Anton Morozov, JHEP 12, 116 (2012). arXiv:1211.4596

17. A. Morozov, JETP Lett. (2013). arXiv: 1208.3544

18. H. Itoyama, A. Mironov, A. Morozov, A. Morozov, IJMP A28, 1340009 (2013). arXiv:1209.6304

19. N. Yu, V.G.Turaev Reshetikhin, Comm. Math. Phys. 127, 1-26 (1990)

20. E. Guadagnini, M. Mintchev, M. Martellini, In Clausthal 1989, Proceedings, Quantum groups, pp. 307-317 (1989)

21. E. Guadagnini, M. Martellini, M. Mintchev, Phys. Lett. B 235, 275 (1990)

22. V.G. Turaev, O.Y. Viro, Topology 31, 865 (1992)

23. A. Morozov, A. Smirnov, Nucl. Phys. B 835, 284-313 (2010). arXiv: 1001.2003

24. A. Smirnov, Proc. of International School of Subnuclar Phys. Erice, Italy (2009). arXiv:0910.5011

25. R.K. Kaul, T.R. Govindarajan, Nucl. Phys. B 380, 293-336 (1992). arXiv:hep-th/9111063

26. R.K. Kaul, T.R. Govindarajan, ibid. B 393, 392-412 (1993)

27. P. Ramadevi, T.R. Govindarajan, R.K. Kaul, Nucl. Phys. B 402, 548-566 (1993). arxiv:hep-th/9212110

28. P. Ramadevi, T.R. Govindarajan, R.K. Kaul, Nucl. Phys. B 422, 291-306 (1994). arXiv:hep-th/9312215

29. P. Ramadevi, T.R. Govindarajan, R.K. Kaul, Mod. Phys. Lett. A 10, 1635-1658 (1995). arXiv:hep-th/9412084

30. P. Ramadevi, T. Sarkar, Nucl. Phys. B 600, 487-511 (2001). arXiv:hep-th/0009188

31. P. Borhade, P. Ramadevi, T. Sarkar, Nucl. Phys. B 678, 656-681 (2004). arXiv:hep-th/0306283

32. P. Ramadevi, Zodinmawia. arXiv:1107.3918, arXiv:1209.1346

33. S. Nawata, P. Ramadevi, Lett Zodinmawia, Math. Phys. 103, 13891398 (2013). arXiv:1302.5143

34. A. Mironov, A. Morozov, A. Morozov, In: A. Rebhan, L. Katzarkov, J. Knapp, R. Rashkov, E. Scheidegger, Eds. Strings, gauge fields, and the geometry behind: the legacy of Maximilian Kreuzer. World Scientific, pp. 101-118 (2013). arXiv:1112.5754
35. A. Mironov, A. Morozov, A. Morozov, JHEP 03, 034 (2012). arXiv: 1112.2654

36. H. Itoyama, A. Mironov, A. Morozov, A. Morozov, IJMP A27, 1250099 (2012). arXiv: 1204.4785

37. A. Anokhina, A. Mironov, A. Morozov, A. Morozov, Nucl. Phys. B 868, 271-313 (2013). arXiv:1207.0279

38. A. Anokhina, A. Mironov, A. Morozov, A. Morozov, Adv. High Energy Phys. 2013, 931830 (2013). arXiv: 1304.1486

39. A. Anokhina, A. Morozov, Theor. Math. Phys. 178, 1-58 (2014). arXiv: 1307.2216

40. A. Anokhina. arXiv: 1412.8444

41. D. Galakhov, D. Melnikov, A. Mironov, A. Morozov, A. Sleptsov, Phys. Lett. B 743, 71-74 (2015). arXiv:1412.2616

42. A. Mironov, A. Morozov, A. Sleptsov, JHEP 07, 069 (2015). arXiv: 1412.8432

43. S. Nawata, P. Ramadevi, V.K. Singh. arXiv: 1504.00364

44. A. Mironov, A. Morozov, A. Morozov, P. Ramadevi, V.K. Singh, JHEP 1507, 109 (2015). arXiv:1504.00371

45. A. Mironov, A. Morozov, Nucl. Phys. B 899, 395-413 (2015). arXiv: 1506.00339

46. A. Mironov, A. Morozov, A. Morozov, A. Sleptsov, J. Mod. Phys. A30, 1550169 (2015). arXiv:1508.02870

47. A. Mironov, A. Morozov, A. Morozov, P. Ramadevi, V.K. Singh, A. Sleptsov. arXiv: 1601.04199

48. A. Mironov, A. Morozov, A. Morozov, A. Sleptsov. arXiv: 1605.02313

49. A. Mironov, A. Morozov, A. Morozov, A. Sleptsov. arXiv:1605.03098

50. A. Mironov, A. Morozov, A. Morozov, A. Sleptsov, Phys. Lett. B 760, 45-58 (2016). arXiv: 1605.04881

51. A. Morozov, Nucl. Phys. B 911, 582-605 (2016). arXiv:1605.09728

52. A. Morozov, JHEP 1609, 135 (2016). arXiv: 1606.06015

53. Y. Kononov, A. Morozov. arXiv: 1609.00143

54. A. Mironov, R. Mkrtchyan, A. Morozov, JHEP 02, 78 (2016). arXiv: 1510.05884

55. A. Mironov, A. Morozov, Phys. Lett. B 755, 47-57 (2016). arXiv:1511.09077

56. I. Tuba, H. Wenzl, math/9912013

57. D. Galakhov, A. Mironov, A. Morozov, JETP 120 (2015) 623-663 (ZhETF, 147 (2015) 623-663). arXiv:1410.8482

58. D. Galakhov, D. Melnikov, A. Mironov, A. Morozov, Nucl. Phys. B 899, 194-228 (2015). arXiv:1502.02621 